\title{
A Correlative Microscopy Study of the Nanostructured Surface of Lotus Leaf
}

\author{
K. Khan*, A. Calabro*, C. Queenan*, D. Becker*, J. Morales**, A. Subramaniam* \\ * Bergen County Academies, Nano-Structural Imaging Lab, 200 Hackensack Avenue, Hackensack, \\ NJ 07601 \\ ** City College of New York, Electron Microscopy Facility, 160 Convent Avenue, New York, NY \\ 10031
}

The lotus leaf has become a specimen of interest due to its super-hydrophobic and self-cleaning properties. It is believed that these properties originate from the micro- and nanostructures found on the surface of the lotus leaf. The microstructures, or papillae, cause the trapping of air when in contact with water, which leads to the leaf's super-hydrophobicity [1]. Researchers in both nanotechnology and the textile industry are seeking to mimic the natural structure of the lotus leaf in order to create self-cleaning fabrics and super-hydrophobic surfaces.

To date, studies of lotus leaf and structures with similar properties, such as butterfly wing and rice leaf, have been performed almost exclusively by scanning electron microscopy. For this reason, we chose to conduct a correlative microscopy study to determine if there are any structures in the crosssection of the lotus leaf which contribute to its self-cleaning properties.

For SEM imaging, small squares (2mm x 2mm) were trimmed from the lotus leaf, fixed with 5\% glutaraldehyde in PBS, dehydrated in a gradient series of ethanol, critical point dried and mounted on aluminum SEM stubs with double-sided conductive carbon tape. For TEM imaging, similar sized squares were trimmed from the lotus leaf, fixed with $4 \%$ glutaraldehyde, $2 \%$ formaldehyde in $0.1 \mathrm{M}$ sodium cacodylate buffer, $\mathrm{pH} 7.4$, post-fixed in $2 \%$ buffered osmium tetroxide, dehydrated in a gradient series of acetone, embedded in an epoxy resin (EMBed 812, Electron Microscopy Sciences) and cured for 48 hours in a $60^{\circ} \mathrm{C}$ oven. Blocks were then trimmed, sectioned on an ultramicrotome and placed on 200 mesh copper TEM grids. Sections were stained with $4 \%$ uranyl acetate for 2 hours followed by $0.5 \%$ lead citrate for 15 minutes.

SEM analysis (Figure 1) of the lotus leaf confirmed structures previously published: micron-scale papillae distributed randomly on the surface of the leaf. Tilting the sample to an angle of $20^{\circ}-30^{\circ}$ aided in determining the 3-dimensional structure of the papillae.

TEM analysis (Figure 2) of the lotus papillae structures revealed layers of material surrounding a central core composed of cellular material and some air space. The most notable feature is the outermost layer of fibrous material. These nanofibers may contribute to the super-hydrophobic properties of the lotus leaf.

\section{References}

[1] Taolei Sun et al., Acc. Chem. Res. (2005) 38 644-652.

[2] The authors would like to acknowledge all of the students who participated in the Nanotechnology Summer Research Program, 2009 for their assistance in acquiring the electron micrographs. 

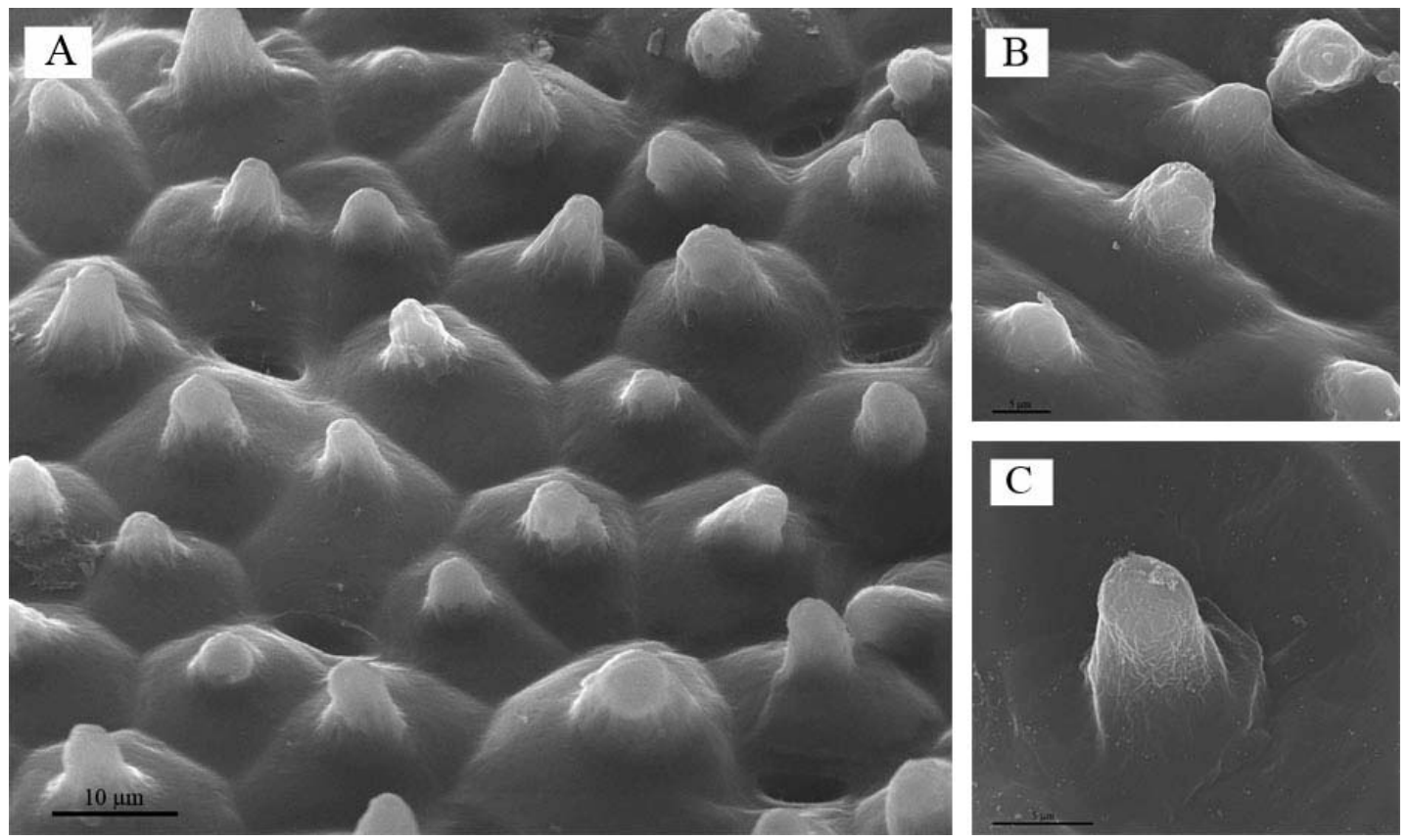

1. SEM image of the surface structure of Lotus leaf with $20^{\circ}$ of tilt. Image A is a low magnification image of the leaf surface; Image B is increased magnification and Image $\mathrm{C}$ is an individual papilla. All images acquired on FEI Quanta $2003 \mathrm{D}$ at $30 \mathrm{kV}$.
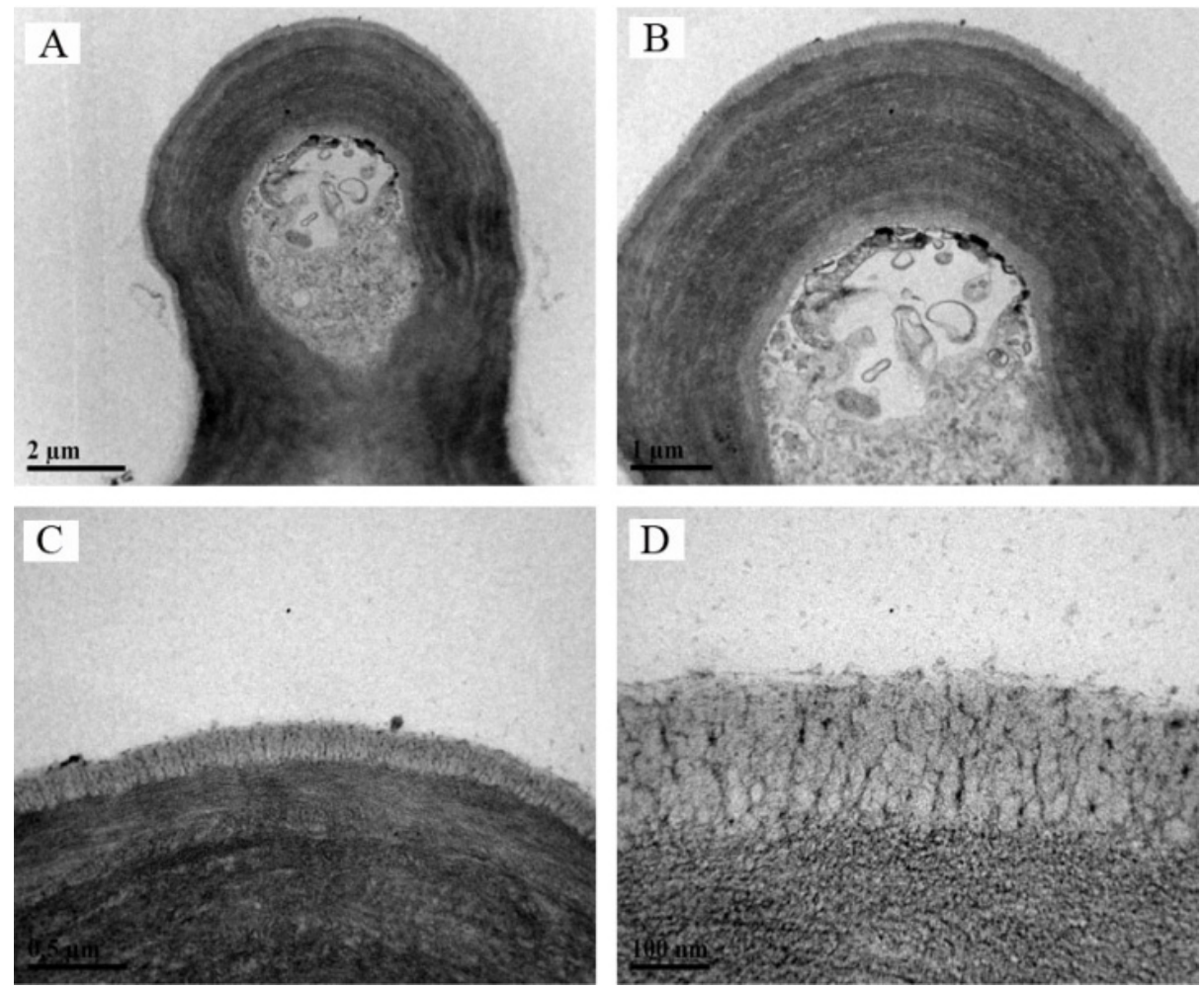

Figure 2. TEM images of an individual papilla. Image A is entire papilla; Images B-D are increasing magnification of the same area. Images $\mathrm{C}-\mathrm{D}$ reveal unique layers of material, including fibrous external layer about $150 \mathrm{~nm}$ in thickness. All images acquired on a JEOL JEM 2100 at 200 $\mathrm{kV}$. 